\title{
Peptidomics Analysis of Virulent Peptides Involved in Streptococcus suis Pathogenesis
}

\author{
Chadaporn Chaiden ${ }^{1}$, Janthima Jaresitthikunchai ${ }^{2}$, Narumon Phaonakrop ${ }^{2}$, Sittiruk Roytrakul ${ }^{2, *}$, \\ Anusak Kerdsin ${ }^{3}$ (D) and Suphachai Nuanualsuwan ${ }^{1,4, *}$ \\ 1 Department of Veterinary Public Health, Faculty of Veterinary Sciences, Chulalongkorn University, \\ Bangkok 10330, Thailand; tiny_my_mild@outlook.com \\ 2 Functional Proteomics Technology Laboratory, Functional Ingredients and Food Innovation Research Group, \\ National Center for Genetic Engineering and Biotechnology, National Science and Technology for \\ Development Agency, Pathum Thani 12120, Thailand; janthima.jar@biotec.or.th (J.J.); \\ narumon.pha@biotec.or.th (N.P.) \\ 3 Faculty of Public Health, Kasetsart University Chalermphrakiat Sakon Nakhon Province Campus, \\ Sakon Nakhon 47000, Thailand; anusak.ke@ku.th \\ 4 Food Risk Hub, Research Unit of Chulalongkorn University, Bangkok 10330, Thailand \\ * Correspondence: sittiruk@biotec.or.th (S.R.); suphachai.n@chula.ac.th (S.N.)
}

Citation: Chaiden, C;

Jaresitthikunchai, J.; Phaonakrop, N.; Roytrakul, S.; Kerdsin, A.; Nuanualsuwan, S. Peptidomics Analysis of Virulent Peptides Involved in Streptococcus suis Pathogenesis. Animals 2021, 11, 2480. https://doi.org/10.3390/ani11092480

Received: 8 July 2021

Accepted: 22 August 2021

Published: 24 August 2021

Publisher's Note: MDPI stays neutral with regard to jurisdictional claims in published maps and institutional affiliations.

Copyright: (c) 2021 by the authors. Licensee MDPI, Basel, Switzerland. This article is an open access article distributed under the terms and conditions of the Creative Commons Attribution (CC BY) license (https:// creativecommons.org/licenses/by/ $4.0 /)$.
Simple Summary: The virulence factors and pathogenesis of S. suis are inconclusive. Here, the associated proteins, or their derived peptides, involved in the survival of $S$. suis when simulated with a blood environment are demonstrated. The results reveal the derived peptides or proteins of $S$. suis potentially serving as the putative virulence factors. Further studies based on our findings could be used to fulfill the knowledge gap of $S$. suis pathogenesis.

Abstract: Streptococcus suis (S. suis) is a zoonotic pathogen causing severe streptococcal disease worldwide. S. suis infections in pigs and humans are frequently associated with the virulent $S$. suis serotype 2 (SS2). Though various virulence factors of $S$. suis have been proposed, most of them were not essentially accounted for in the experimental infections. In the present study, we compared the peptidomes of highly virulent SS2 and SS14 in humans, the swine causative serotypes SS7 and SS9, and the rarely reported serotypes SS25 and SS27, and they were cultured in a specified culture medium containing whole blood to simulate their natural host environment. LC-MS/MS could identify 22 unique peptides expressed in the six $S$. suis serotypes. Under the host-simulated environment, peptides from the ABC-type phosphate transport system (SSU05_1106) and 30S ribosomal protein S2 (rpsB) were detected in the peptidome of virulent SS2 and SS14. Therefore, we suggest that these two proteins or their derived peptides might be involved in the survival of $S$. suis when simulated with a blood environment.

Keywords: peptidomics analysis; virulent peptides; Streptococcus suis; pathogenesis; virulence factor; zoonosis

\section{Introduction}

Streptococcus suis is a zoonotic bacterium causing severe streptococcal infection in both humans and pigs. Among 35 serotypes classified by the immunogenics of their capsular polysaccharides [1], SS serotype 2 (SS2) is the most prevalent, followed by SS14, the causative agent of $S$. suis zoonotic infection in humans and pigs worldwide [2,3]. For swine infections, SS7 and SS9 are associated with the clinical swine cases in the European region while SS3 is usually disseminated in North America and Asia [2]. The clinical symptoms of $S$. suis infection range from a common bacterial infection to streptococcal toxic shocklike syndrome (TSST) [1,4]. Although some virulence factors of $S$. suis such as capsular polysaccharide (CPS), suilysin, muramidase-released protein (MRP), and extracellular 
factors have been described [5-7], a thorough list of virulence factors of S. suis particularly from some other serotypes also needs to be explored. Proteomic and peptidomic techniques have been widely adopted and used to investigate the microbial proteomes for the virulence factor, diagnostic marker, and vaccine targets $[8,9]$. In the present study, a high-performance liquid chromatography-mass spectrometry/mass spectrometry method (LC-MS/MS) was used to determine the peptidomes of the highly virulent to humans SS2 and SS14, swine causative serotypes SS7 and SS9, and rarely reported serotypes SS25 and SS27 cultured in a specified culture medium containing whole blood, simulating their natural host environment. This study aimed to explore some putative virulence factors involved in the survival of $S$. suis in the blood environment.

\section{Methods}

\subsection{Bacterial Strains}

Six reference $S$. suis serotypes, originating from diseased pigs, were included in the present study. The virulent S. suis to humans were SS2 (ATCC 700794) and SS14 (13730) and the non-virulent S. suis to humans were SS7 (8074), SS9 (22083), SS25 (893576-3), and SS27 (89-5259). S. suis bacteria were cultured on Columbia blood agar (Difco Laboratories, Detroit, MI, USA) with $5 \%(v / v)$ sheep's blood at $37^{\circ} \mathrm{C}$ in anaerobic conditions using a GasPak Anaerobic System (Mitsubishi Gas Chemical Co., Inc., Tokyo, Japan) for $24 \mathrm{~h}$. Sequencing of the 16S rRNA gene was performed to validate their strains [10]. GenBank accession numbers of the 16S rRNA gene of SS2, SS14, SS7, SS9, SS25 and SS27 are LS483418.1, AF009489.1, AF009482.1, AF009484.1, AF009500.1 and AF009502.1, respectively. The bacterial colonies were then cultured in a Todd-Hewitt broth (THB) (Difco Laboratories, Detroit, MI, USA) and preserved with $20 \%$ glycerol at $-80{ }^{\circ} \mathrm{C}$ for further study.

\subsection{Preparation of Peptidome}

SS2, SS14, SS7, SS9, SS25, and SS27 were independently cultured in THB at $37^{\circ} \mathrm{C}$ for $18 \mathrm{~h}$. A mixture of $200 \mu \mathrm{L}$ of cultured bacterium $\left(\cong 10^{7} \mathrm{CFU} / \mathrm{mL}\right)$ of the $S$. suis serotype and $800 \mu \mathrm{L}$ of fresh whole sheep's blood (red blood cells $\cong 10^{6}$ cells $/ \mathrm{mL}$ ) was incubated at $37^{\circ} \mathrm{C}$ for $30 \mathrm{~min}$. The results of an MTT (3-[4,5-dimethylthiazol-2-yl]-2,5-diphenyltetrazolium bromide) assay [11] revealed that after $30 \mathrm{~min}$ of incubation time with whole blood, the optical density at $570 \mathrm{~nm}$ significantly rose $(p<0.01)$, representing the proliferation of $S$. suis viable cells (data not shown). We then assumed that virulence factors were triggered as part of the S. suis pathogenicity during the bacterial colonization in the host in as soon as $30 \mathrm{~min}$ of exposure to the blood culture medium. This procedure was repeated five times for each S. suis serotype. The bacterial peptides were extracted from the $S$. suis bacterial suspension according to a previous study [12]. Briefly, after centrifugation at $300 \mathrm{~g}$ for $5 \mathrm{~min}, 5 \%(v / v)$ trifluoroacetic acid (TFA) in absolute acetonitrile (ACN) was added to the pellet and the suspension was dissolved by gentle vortexing. The samples were dried to remove $\mathrm{ACN}$ and resuspended with $0.1 \%(v / v)$ formic acid. A Lowry assay was used to determine peptide concentration [13].

\section{3. $L C-M S / M S$}

An HCTUltra PTM Discovery System (Bruker Daltonics Ltd., Bremen, Germany) coupled with an UltiMate 3000 LC System (Dionex Ltd., Camberley, UK) was used to analyze peptides in each sample. The peptide samples were separated on a nanocolumn (Acclaim PepMap 100 column $75 \mathrm{um} \times 5 \mathrm{~cm}$ ) using reversed-phase high-performance liquid chromatography. Two eluents were used. Eluent $\mathrm{A}$ was $0.1 \%$ formic acid and eluent $\mathrm{B}$ was $80 \% \mathrm{ACN}$ in water containing $0.1 \%$ formic acid. A 5-55\% eluent B gradient was used to elute peptides at a constant flow rate of $0.30 \mu \mathrm{L} / \mathrm{min}$ for $30 \mathrm{~min}$. Electrospray ionization was carried at $1.6 \mathrm{kV}$ using the CaptiveSpray. Nitrogen, a drying gas, was applied with a flow rate of about $50 \mathrm{~L} / \mathrm{h}$. Collision-induced-dissociation product ion mass spectra were achieved using nitrogen gas as the collision gas. Mass spectra (MS) and MS/MS spectra were collected in positive-ion mode at $2 \mathrm{~Hz}$ over the $(\mathrm{m} / \mathrm{z})$ range $150-2200$. The collision 
energy was adjusted to $10 \mathrm{eV}$ as a function of the $m / z$ value. The LC-MS analysis of each sample was performed in triplicate.

\subsection{Peptidomic Data Analysis}

DeCyder MS Differential Analysis Software (DeCyderMS, GE Healthcare) was used to quantitate peptides from MS/MS signal data. ANOVA statistical analysis, incorporated into the DeCyder MS Differential Analysis Software was used to analyze significantly different peptide peaks. The analyzed MS/MS data were submitted to identify proteins against the Uniprot database using the Mascot software (Matrix Science, London, UK). The parameters for the database search were taxonomy (Streptococcus suis), enzyme (NoCleave), variable modifications (oxidation of methionine residues), mass values (monoisotopic), protein mass (unrestricted), peptide mass tolerance (1.2 Da), fragment mass tolerance ( $\pm 0.6 \mathrm{Da})$, peptide charge state $(1+, 2+$ and $3+)$, and missed cleavages (3). The MS/MS spectra were manually inspected for the full sequence of the main fragmentation series by Mascot. All differentially expressed peptides were analyzed for their intersections among the different sample groups using jvenn [14]. Information about particular proteins was used in the annotation by UniProtKB/Swiss-Prot entries (http: / / www.uniprot.org/ accessed on 29 July 2020). The relationships between identified proteins (from S. suis) and other interesting molecules were investigated using STITCH 5.0 (http:/ / stitch.embl.de/ accessed on 24 August 2020) [15].

\section{Results}

\section{Peptidomic Analysis by LC-MS/MS}

The different expressions of $S$. suis peptides cultured using the THB medium and the THB medium containing whole blood were determined using LC-MS/MS. A total of 1919 peptides were identified in the peptidome of SS2. All 1919 peptides were significantly identified using the DeCyder MS Differential Analysis software $(p<0.05)$. The distributions of peptides of SS2 cultivated in certain conditions were shown in Figure 1. Only peptides found in SS2 grown in the THB medium supplemented with the whole blood (359 peptides) were used for further investigation (Figure 1). Likewise, peptides identified in SS14, SS7, SS9, SS25, and SS27 were included for further investigation. The complete list of peptides for all six serotypes was provided in the Supplementary Materials.

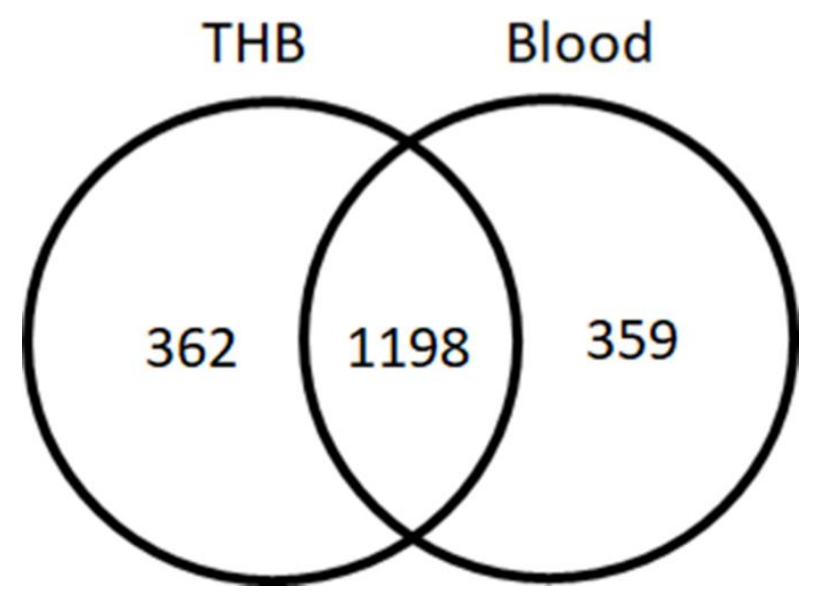

Figure 1. Venn diagram showing the number of proteins in SS2 cultivated in the THB medium with and without whole blood.

Overall, 22 peptides derived from SS2, SS14, SS7, SS9, SS25, and SS27 proteins with known functions, matched in the STITCH database, were selected and are shown in Table 1. In particular, three peptides were identified only in virulent $S$. suis. Interestingly, out of three peptides, two of them were consistently detected in virulent $S$. suis serotype 2 and/or serotype 14 including the ABC-type phosphate transport system (SSU05_1106) 
and $30 \mathrm{~S}$ ribosomal protein S2 (rpsB), whereas 12 peptides were expressed only in nonvirulent S. suis SS7, SS9, SS25, and SS27 (Table 2). They were transcriptional regulator (SSU05_2039), $\alpha-1,4$ glucan phosphorylase, PTS system ascorbate-specific transporter subunit IIC (SSU05_2063), branched-chain amino acid permease (SSU05_0780), $10 \mathrm{kDa}$ chaperonin (GroS), low-temperature requirement A (SSU05_0642), Cps2J (cps2J), truncated MRP, hypothetical protein (SSU05_1457), hypothetical protein (SSU05_0461), hypothetical protein (SSU05_1869) and a hypothetical protein (SSU05_0141).

Table 1. Identified peptides derived from S. suis proteins with known function cultured under the host simulated environment.

\begin{tabular}{|c|c|c|}
\hline Gene Locus & Annotated Protein Function (Amino-Acid Length) & Function or Subcellular Location \\
\hline SSU05_2116 & $5^{\prime}$-nucleotidase $/ 2^{\prime}, 3^{\prime}$-cyclic phosphodiesterase and related esterases (448) & nucleotide catabolic activity \\
\hline SSU05_2039 & transcriptional regulator (149) & DNA-binding transcription factor activity \\
\hline $\mathrm{N} / \mathrm{A}$ & $\alpha-1,4$ glucan phosphorylase $(755)$ & carbohydrate metabolism \\
\hline SSU05_1489 & K01223 6-phospho-beta-glucosidase [EC-3.2.1.86] (474) & carbohydrate metabolism \\
\hline SSU05_0392 & $4-\alpha$-glucanotransferase (364) & carbohydrate metabolism \\
\hline SSU05_1803 & 3-oxoacyl-[acyl-carrier-protein] reductase (244) & fatty acid biosynthesis \\
\hline SSU05_2063 & PTS system ascorbate-specific transporter subunit IIC (482) & cell membrane/membrane \\
\hline SSU05_1254 & ABC transporter permease (197) & transmembrane transporter \\
\hline SSU05_1106 & ABC-type phosphate transport system (83) & periplasmic component \\
\hline SSU05_0780 & branched-chain amino acid permease (188) & cell membrane/membrane \\
\hline$g m k$ & guanylate kinase (216) & GMP recycling \\
\hline tet $\mathrm{M}$ & TetM (639) & response to antibiotic \\
\hline gros & $10 \mathrm{kDa}$ chaperonin (102) & ATPase activity \\
\hline SSU05_0642 & putative low-temperature requirement A (371) & transmembrane protein \\
\hline cps $2 \mathrm{~J}$ & Cps2J (332) & capsular biosynthesis \\
\hline mrp & truncated MRP (1261) & secreted protein \\
\hline$r p s \mathrm{~B}$ & 30 S ribosomal protein S2 (258) & ribosomal protein \\
\hline SSU05_1457 & hypothetical protein (91) & \\
\hline SSU05_0461 & hypothetical protein (567) & \\
\hline SSU05_1869 & hypothetical protein (178) & \\
\hline SSU05_0141 & hypothetical protein (318) & \\
\hline SSU05_1991 & hypothetical protein (412) & \\
\hline
\end{tabular}

A peptide $5^{\prime}$-nucleotidase $/ 2^{\prime}, 3^{\prime}$-cyclic phosphodiesterase and related esterases (SSU05_2116), associated with nucleotide catabolic activity, was detected in SS2, SS14, and SS9, while the transcriptional regulator protein (SSU05_2039) was found in SS7 and SS25. Three peptides related to carbohydrate metabolism were identified, including; K01223 6-phospho-betaglucosidase (SSU05_1489) in SS2, SS14, and SS9, $\alpha-1,4$ glucan phosphorylase in SS9 and SS25, and 4- $\alpha$-glucanotransferase (SSU05_0392) in SS14. Moreover, a peptide of fatty acid biosynthesis protein, 3-oxoacyl-[acyl-carrier-protein] reductase (SSU05_1803) was also identified in SS2, SS14, SS7, and SS27.

Peptides involved in the membrane of the bacteria were also detected, consisting of a derived peptide of the PTS system ascorbate-specific transporter subunit IIC (SSU05_2063) in SS7 and SS9, ABC transporter permease (SSU05_1254) in SS14 and SS7, and branchedchain amino acid permease (SSU05_0780) in SS7.

Other peptides were guanylate kinase (gmk) expressed in SS2, SS14, SS9, SS25, and SS27, and tetracycline resistance protein (TetM) was identified in SS14, SS25, and SS27. Peptide co-chaperonin GroS was found in SS7. A putative low-temperature requirement A protein (SSU05_0642) was found in SS7. Furthermore, Cps2J was identified in SS25, and a truncated MRP was found in SS7.

Various peptides belonged to a hypothetical group, such as SSU05_1457 hypothetical protein in SS25, SSU05_0461 hypothetical protein in SS27, SSU05_1869 hypothetical protein in SS25, and SS27, SSU05_0141 hypothetical protein in SS25, and SSU05_1991 hypothetical protein in SS2, SS7, SS25, and SS27. 
Table 2. Distribution of 22 identified peptides derived from S. suis proteins of virulent vs. non-virulent S. suis to human cultured under host simulated environment.

\begin{tabular}{|c|c|c|c|c|c|c|}
\hline \multirow{3}{*}{ Identified Protein } & \multicolumn{6}{|c|}{ S. suis Serotype } \\
\hline & \multicolumn{2}{|c|}{ Virulent } & \multicolumn{4}{|c|}{ Non-Virulent } \\
\hline & 2 & 14 & 7 & 9 & 25 & 27 \\
\hline $5^{\prime}$-nucleotidase $/ 2^{\prime}, 3^{\prime}$-cyclic phosphodiesterase and related esterases & + & + & & + & & \\
\hline transcriptional regulator & & & + & & + & \\
\hline$\alpha-1,4$ glucan phosphorylase & & & & + & + & \\
\hline K01223 6-phospho-beta-glucosidase [EC-3.2.1.86] & + & + & & + & & \\
\hline 4 - $\alpha$-glucanotransferase & & + & & & & \\
\hline 3-oxoacyl-[acyl-carrier-protein] reductase & + & + & + & & & + \\
\hline PTS system ascorbate-specific transporter subunit IIC & & & + & + & & \\
\hline $\mathrm{ABC}$ transporter permease & & + & + & & & \\
\hline ABC-type phosphate transport system & + & + & & & & \\
\hline branched-chain amino acid permease & & & + & & & \\
\hline guanylate kinase & + & + & & + & + & + \\
\hline TetM & & + & & & + & + \\
\hline $10 \mathrm{kDa}$ chaperonin & & & + & & & \\
\hline putative low-temperature requirement $\mathrm{A}$ & & & + & & & \\
\hline Cps2J & & & & & + & \\
\hline truncated MRP & & & + & & & \\
\hline 30S ribosomal protein S2 & + & + & & & & \\
\hline SSU05_1457 hypothetical protein & & & & & + & \\
\hline SSU05_0461 hypothetical protein & & & & & & + \\
\hline SSU05_1869 hypothetical protein & & & & & + & + \\
\hline SSU05_0141 hypothetical protein & & & & & + & \\
\hline SSU05_1991 hypothetical protein & + & & + & & + & + \\
\hline Sum & 7 & 9 & 9 & 5 & 9 & 6 \\
\hline
\end{tabular}

\section{Discussion}

S. suis pathogenesis is triggered by exposure to a certain condition in the host environment [16]. In the present study, six S. suis serotypes were simulated under the human cardiovascular system and they interacted with erythrocytes, leukocytes, and other blood components particularly during bacterial septicemia (bacteremia). A large number of peptides were identified in the peptidome of $S$. suis. Among peptides expressed by reacting with the whole blood, only 22 unique peptides derived from S. suis proteins with known functions, matched in the STITCH database, were selected (Table 1). Interestingly, derived peptides of the ABC-type phosphate transport system and 30S ribosomal protein S2 were simultaneously co-expressed in the highly virulent SS2 and virulent SS14 cultured under the host-simulated condition. Notably, the ABC transporter protein has been reported in the pathogenicity of S. pneumoniae [17], while 4- $\alpha$-glucanotransferase (malQ gene), responsible for starch metabolism in S. mutans, was expressed uniquely in the virulent SS14 [18]. Therefore, derived peptides of the ABC-type phosphate transport system, $30 \mathrm{~S}$ ribosomal protein S2 and 4- $\alpha$-glucanotransferase might serve as specific virulence factors of SS2 and SS14.

The association of $5^{\prime}$-nucleotidase $/ 2^{\prime}, 3^{\prime}$-cyclic phosphodiesterase and related esterases (SSU05_0456) with S. suis has been rarely mentioned to the best of our knowledge. However, $5^{\prime}$-nucleotidase has been considered as a virulence factor in many pathogens including $S$. suis [19-22], because this enzyme is commonly involved in adenosine synthesis. Adenosine, an immunomodulatory molecule, reduces the phagocytic activity of macrophages and also impairs the neutrophil degranulation thus decreasing the inflammatory responses of the host immune system [19]. A previous study by Liu et al., (2014) demonstrated that $5^{\prime}$-nucleotidase is an essential factor for $S$. suis escaping from the host's innate immunity as the enzyme-deficient mutant strain of $S$. suis lost its infectivity in the pig model [20]. Thus, our result was in concordance with Liu et al., (2014) as a derived peptide of $5^{\prime}$-nucleotidase 
was found in the virulent SS2 and SS14 reacting with the blood in the host-simulated environment. This emphasized the significance of the survival proteins and/or peptides initially expressed to tackle the host-simulated immunity.

The $\alpha-1,4$ glucan phosphorylase, K01223 6-phospho- $\beta$-glucosidase [EC-3.2.1.86] and the 4- $\alpha$-glucanotransferase associated with carbohydrate metabolism were exhibited in SS9 and SS25; SS2, SS14, and SS9; and SS14, respectively. These results are compatible with a study by Zhang et al., (2014) that identified 6-phospho- $\beta$-glucosidase in two Chinese isolates of virulent SS2 [23]. Unfortunately to the best of our knowledge, the direct association of these three enzymes with S. suis is not evident. However, the carbohydrate availability is positively correlated with the virulence of $S$. suis using the host cell invasion i.e., the adequate carbohydrate supply upregulates the virulence gene regulation of virulent $S$. suis as described previously in a transcriptomic study [24].

The biosynthesis of fatty acids is important for the pathogenicity and adaptation of bacteria in the host environment since fatty acids serve as an energy source and a material for cell-wall synthesis. In addition, the pattern of the lipid cell walls also acts as a factor modulating the immune responses of the host $[25,26]$. In the present study, a derived peptide of 3-oxoacyl-[acyl-carrier-protein] reductase responsible for the fatty acid biosynthesis was identified in SS2, SS14, SS7, and SS27. The previous studies demonstrated that this enzyme was associated with the fatty acid biosynthesis of virulent SS2 [27,28]. Thus, we could assume that this fatty acid biosynthesis-associated enzyme is significant for S. suis adaptation in the host-simulated environment.

Derived peptides of the $\mathrm{ABC}$ transporter permease and the $\mathrm{ABC}$-type phosphate transport system were found in the peptidomes of SS14 and SS7, and SS2 and SS14, respectively. Generally, $\mathrm{ABC}$ transporter proteins play an important role in the transportation of various substrates including nutrients for the biosynthesis of the bacterial cell. Some of the ABC transporters serve as efflux pumps. Previous studies reported that the increased expression of an $\mathrm{ABC}$ efflux pump was related to fluoroquinolones resistance in S. pneumoniae and $S$. suis $[29,30]$. As shown in Figure 2, the networks of protein-protein interactions analyzed by the STITCH program, version 5.0, demonstrated high associations of the ABC-type phosphate transport system (SSU05_1106) with other phosphate ABC transporters (edge confidence score 0.900 ), responsible for phosphate import. Furthermore, these proteins are associated with the biofilm formation of SS2 [31] and are required for the disease pathogenesis of S. pneumoniae [17]. The branched-chain amino acid permease, one of the ABC transporter proteins, is involved in the transport of leucine, isoleucine, and valine [32]. A previous report also suggested that the branched-chain amino acid permease was related to the pathogenicity of some bacteria [17]. A certain role for the $A B C$ transporter as a virulence factor of bacteria is still not clear. However, the presence of $A B C$ transporters in the virulent to humans SS2, SS14, and non-virulent to humans SS7 indicated that the translocation of substrates particularly phosphate and some amino acids (leucine, isoleucine, and valine) using these $A B C$ transporter proteins is perhaps required for the survival and then the pathogenesis (pathway) of virulent $S$. suis in the host-simulated environment.

Guanylate kinase phosphorylates the guanosine monophosphate to guanosine diphosphate, a precursor of the guanosine triphosphate required for the transcription of RNA synthesis. This enzyme was inhibited when the bacteria were forced to survive in the amino-acid restricted condition [33]. In the present study with adequate nutrients in the host-simulated environment, a peptide of this enzyme was identified in SS2, SS14, SS9, SS25, and SS27. This finding was in line with a previous report indicating that this enzyme is conserved and adaptively expressed depending on the nutrient availability [33]. 


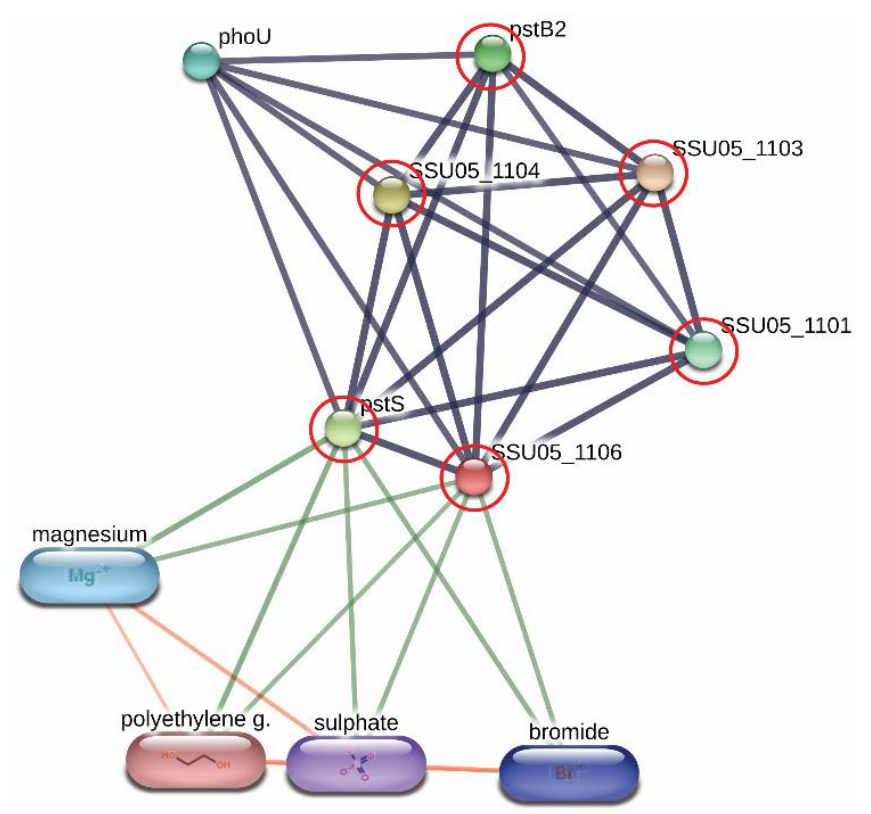

Figure 2. Relationship between ABC-type phosphate transport system (SSU05_1106) and other phosphate ABC transporters; SSU05_1101, SSU05_1103, SSU05_1104, pstS and pstB2 involved in phosphate import from STITCH 5.0 software. The grey thick lines represent the highest edge confidence scores (0.900) indicating the high strength of the protein interactions at the functional level.

The tetracycline-resistance gene, tet $\mathrm{M}$, encoded TetM protein responsible for the inhibitory effect of tetracycline, is distributed among virulent S. suis. [34-36]. An outbreak report in China demonstrated that the tetracycline-resistance strains were associated with S. suis infections in both pigs and humans [34]. Our results revealed the expression of the TetM protein in SS14, SS25, and SS27 cultured under a host-simulated environment. This finding is in concordance with a previous report indicating that $S$. suis carried and spread the tet $\mathrm{M}$ gene among $\mathrm{S}$. suis strains [34].

The association of S. suis and $10 \mathrm{kDa}$ chaperonin is rarely reported. This protein is specified as a protein folding facilitator and also a virulence factor of bone resorption in Mycobacterium tuberculosis infection [37]. In the present study, a peptide derived from $10 \mathrm{kDa}$ chaperonin was detected in the peptidome of SS7.

So far, the CPS of $S$. suis has been mentioned as an important virulence factor of S. suis [7]. The cps gene cluster facilitates the regulation of CPS synthesis. The Cps2J is essential for CPS biosynthesis because the deletion mutant of $\Delta c p s 2 \mathrm{~J}$ results in the imperfection of CPS [38]. The presence of Cps2J only in SS25 might explain why the bacterium was likely to synthesize CPS to protect itself from phagocytosis by the host immune cells [7]. Cps2J was identified in SS14 and SS25 while Cps2A and Cps2C were identified in SS2, SS14, SS9, and SS25 (data not shown).

Previously, MRP has been considered as a virulent marker of S. suis [39]. The mutant strain of $S$. suis demonstrated its virulence even when the MRP protein was deficient. Thus, MRP is more likely to be the virulent maker than the virulence factor. This protein was proposed to be coincidentally expressed together with the true virulence factor [16]. In this study, we identified a peptide derived from truncated MRP only in SS7 under the host-simulated environment. The limited existence of this protein might be explained by the fact that MRP is a cell-wall anchored protein and is released into the supernatant part of the culture medium. Our peptide preparation method did not precipitate proteins in the supernatant thus decreasing the chance of capturing MRP in S. suis cells. A previous study focusing on proteomic analysis of SS2 human isolates also reported no expression of MRP either [40].

Other unique proteins identified in this study were putative low-temperature requirement A protein (SSU05_0642), transcriptional regulator (SSU05_2039), and 30S ribosomal 
protein S2(rpsB). Although the related information regarding their virulence is not available, the expression of $30 S$ ribosomal protein S2 should not be neglected, since these proteins were exhibited only in virulent SS2 and SS14 culture under the host-simulated environment. The role of these proteins in conjunction with the pathogenesis of virulent $S$. suis deserves further study.

Among five hypothetical proteins presented in this study (Table 1), SSU05_1869 hypothetical protein was previously mentioned as a putative ABC transporter protein [41] and SSU05_1991 hypothetical protein was described as possibly associated with bacterial cell growth and division in S. suis serotype 2 [42]. In the present study, SSU05_1991 hypothetical protein was also exhibited in SS2 and also in SS7, SS25, and SS27. When the functions or subcellular locations of the other three hypothetical proteins SSU05_1457, SSU05_0461, and SSU05_0141 are identified, their roles regarding the pathogenesis of virulent $S$. suis will be disclosed.

\section{Conclusions}

S. suis expressed its virulence factor in the form of proteins or peptides to survive in the host. Our study demonstrated various derived peptides expressed in S. suis SS2, SS14, SS7, SS9, SS25, and SS27 reacting with whole blood. Most unique proteins were associated with the transcriptional process and cellular metabolic processes, thus indicating the adaptation of the bacterial cell during the initial period of incubation time in the host-simulated environment. The peptides of the ABC-type phosphate transport system (SSU05_1106) and $30 \mathrm{~S}$ ribosomal protein S2 (rpsB) were solely expressed in virulent to humans SS2 and SS14 under the host-simulated environment and are proposed as putative virulence factors potentially involved in S. suis pathogenesis. The results of this study apply to the diagnostic marker and vaccine targets [8,9]. However, our results only demonstrated the identified peptides by in vitro simulating environment. Further study by in vivo and gene knockout should be conducted to verify our peptidomic findings.

Supplementary Materials: The following are available online at https://www.mdpi.com/article/10 .3390/ani11092480/s1. Table S1: The list of peptides of SS2; Table S2: The list of peptides of SS14; Table S3: The list of peptides of SS7; Table S4: The list of peptides of SS9; Table S5: The list of peptides of SS25; Table S6: The list of peptides of SS27.

Author Contributions: Conceptualization: S.R. and S.N. Data curation: C.C., J.J., N.P. and S.R. Formal analysis: C.C., J.J., N.P. and S.R. Funding acquisition: S.N. Investigation: C.C., J.J., N.P. and S.R. Methodology: J.J., N.P. and S.R. Resources: A.K. Supervision: J.J. and S.R. Validation: S.N. Visualization: C.C. Writing—original draft: C.C. Writing—review \& editing: C.C., A.K., S.R. and S.N. All authors have read and agreed to the published version of the manuscript.

Funding: This study was supported by The 100th Anniversary Chulalongkorn University Fund for Doctoral Scholarship; The 90th Anniversary of Chulalongkorn University Fund (Ratchadaphiseksomphot Endowment Fund); The Scholarship from the Graduate School, Chulalongkorn University to commemorate the 72nd anniversary of his Majesty King Bhumibol Adulyadej is gratefully acknowledged.

Data Availability Statement: Roytrakul, Sittiruk; Nuanualsuwan, Suphachai; Chaiden, Chadaporn; Phaonakrop, Narumon; Jaresitthikunchai, Janthima (2021): Membrane_Peptidome_mzXML.rar. figshare. Dataset. https: / doi.org/10.6084/m9.figshare.14794899.v1 (accessed on 17 June 2021).

Acknowledgments: We thank Sekkarin Ploypetch and Vilailak Tiyao for their technical assistance.

Conflicts of Interest: The authors declare no conflict of interest. The sponsors had no role in the design, execution, interpretation, or writing of the study.

\section{References}

1. Staats, J.J.; Feder, I.; Okwumabua, O.; Chengappa, M.M. Streptococcus suis: Past and present. Vet. Res. Commun. 1997, 21, 381-407. [CrossRef]

2. Goyette-Desjardins, G.; Auger, J.P.; Xu, J.; Segura, M.; Gottschalk, M. Streptococcus suis, an important pig pathogen and emerging zoonotic agent-an update on the worldwide distribution based on serotyping and sequence typing. Emerg. Microbes Infect. 2014, 3, e45. [CrossRef] 
3. Gajdacs, M.; Nemeth, A.; Knausz, M.; Barrak, I.; Stajer, A.; Mestyan, G.; Melegh, S.; Nyul, A.; Toth, A.; Agoston, Z.; et al. Streptococcus suis: An Underestimated Emerging Pathogen in Hungary? Microorganisms 2020, 8, 1292. [CrossRef]

4. Tang, J.; Wang, C.; Feng, Y.; Yang, W.; Song, H.; Chen, Z.; Yu, H.; Pan, X.; Zhou, X.; Wang, H.; et al. Streptococcal toxic shock syndrome caused by Streptococcus suis serotype 2. PLoS Med. 2006, 3, e151. [CrossRef]

5. Baums, C.G.; Valentin-Weigand, P. Surface-associated and secreted factors of Streptococcus suis in epidemiology, pathogenesis and vaccine development. Anim. Health Res. Rev. 2009, 10, 65-83. [CrossRef]

6. Jacobs, A.A.; Loeffen, P.L.; van den Berg, A.J.; Storm, P.K. Identification, purification, and characterization of a thiol-activated hemolysin (suilysin) of Streptococcus suis. Infect. Immun. 1994, 62, 1742-1748. [CrossRef]

7. Smith, H.E.; Damman, M.; van der Velde, J.; Wagenaar, F.; Wisselink, H.J.; Stockhofe-Zurwieden, N.; Smits, M.A. Identification and characterization of the cps locus of Streptococcus suis serotype 2: The capsule protects against phagocytosis and is an important virulence factor. Infect. Immun. 1999, 67, 1750-1756. [CrossRef]

8. Perez-Llarena, F.J.; Bou, G. Proteomics as a Tool for Studying Bacterial Virulence and Antimicrobial Resistance. Front. Microbiol. 2016, 7, 410. [CrossRef]

9. Wu, H.J.; Wang, A.H.J.; Jennings, M.P. Discovery of virulence factors of pathogenic bacteria. Curr. Opin. Chem. Biol. 2008, 12, 93-101. [CrossRef]

10. Chatellier, S.; Harel, J.; Zhang, Y.; Gottschalk, M.; Higgins, R.; Devriese, L.A.; Brousseau, R. Phylogenetic diversity of Streptococcus suis strains of various serotypes as revealed by $16 \mathrm{~S}$ rRNA gene sequence comparison. Int. J. Syst. Bacteriol. 1998, 48 Pt 2, 581-589. [CrossRef]

11. Van Meerloo, J.; Kaspers, G.J.; Cloos, J. Cell sensitivity assays: The MTT assay. Methods Mol. Biol. 2011, 731, 237-245. [CrossRef]

12. Chaiden, C.; Jaresitthikunchai, J.; Kerdsin, A.; Meekhanon, N.; Roytrakul, S.; Nuanualsuwan, S. Streptococcus suis serotyping by matrix-assisted laser desorption/ionization time-of-flight mass spectrometry. PLoS ONE 2021, 16, e0249682. [CrossRef]

13. Lowry, O.H.; Rosebrough, N.J.; Farr, A.L.; Randall, R.J. Protein measurement with the Folin phenol reagent. J. Biol. Chem. 1951, 193, 265-275. [CrossRef]

14. Bardou, P.; Mariette, J.; Escudie, F.; Djemiel, C.; Klopp, C. jvenn: An interactive Venn diagram viewer. BMC Bioinform. 2014, 15, 293. [CrossRef]

15. Szklarczyk, D.; Santos, A.; von Mering, C.; Jensen, L.J.; Bork, P.; Kuhn, M. STITCH 5: Augmenting protein-chemical interaction networks with tissue and affinity data. Nucleic Acids Res. 2016, 44, D380-D384. [CrossRef]

16. Fittipaldi, N.; Segura, M.; Grenier, D.; Gottschalk, M. Virulence factors involved in the pathogenesis of the infection caused by the swine pathogen and zoonotic agent Streptococcus suis. Future Microbiol. 2012, 7, 259-279. [CrossRef] [PubMed]

17. Basavanna, S.; Khandavilli, S.; Yuste, J.; Cohen, J.M.; Hosie, A.H.; Webb, A.J.; Thomas, G.H.; Brown, J.S. Screening of Streptococcus pneumoniae $\mathrm{ABC}$ transporter mutants demonstrates that LivJHMGF, a branched-chain amino acid ABC transporter, is necessary for disease pathogenesis. Infect. Immun. 2009, 77, 3412-3423. [CrossRef]

18. Sato, Y.; Okamoto-Shibayama, K.; Azuma, T. The malQ gene is essential for starch metabolism in Streptococcus mutans. J. Oral Microbiol. 2013, 5, 21285. [CrossRef]

19. Zheng, L.; Khemlani, A.; Lorenz, N.; Loh, J.M.; Langley, R.J.; Proft, T. Streptococcal 5'-Nucleotidase A (S5nA), a Novel Streptococcus pyogenes Virulence Factor That Facilitates Immune Evasion. J. Biol. Chem. 2015, 290, 31126-31137. [CrossRef]

20. Liu, P.; Pian, Y.; Li, X.; Liu, R.; Xie, W.; Zhang, C.; Zheng, Y.; Jiang, Y.; Yuan, Y. Streptococcus suis adenosine synthase functions as an effector in evasion of PMN-mediated innate immunit. J. Infect. Dis. 2014, 210, 35-45. [CrossRef]

21. Thammavongsa, V.; Kern, J.W.; Missiakas, D.M.; Schneewind, O. Staphylococcus aureus synthesizes adenosine to escape host immune responses. J. Exp. Med. 2009, 206, 2417-2427. [CrossRef]

22. Fan, J.; Zhang, Y.; Chuang-Smith, O.N.; Frank, K.L.; Guenther, B.D.; Kern, M.; Schlievert, P.M.; Herzberg, M.C. Ecto-5'nucleotidase: A candidate virulence factor in Streptococcus sanguinis experimental endocarditis. PLoS ONE 2012, 7, e38059. [CrossRef]

23. Zhang, D.; Du, N.; Ma, S.; Hu, Q.; Lu, G.; Chen, W.; Zeng, C. In vitro transcriptome analysis of two Chinese isolates of Streptococcus suis serotype 2. Genom. Proteom. Bioinform. 2014, 12, 266-275. [CrossRef]

24. Ferrando, M.L.; van Baarlen, P.; Orru, G.; Piga, R.; Bongers, R.S.; Wels, M.; De Greeff, A.; Smith, H.E.; Wells, J.M. Carbohydrate availability regulates virulence gene expression in Streptococcus suis. PLoS ONE 2014, 9, e89334. [CrossRef]

25. Rameshwaram, N.R.; Singh, P.; Ghosh, S.; Mukhopadhyay, S. Lipid metabolism and intracellular bacterial virulence: Key to next-generation therapeutics. Future Microbiol. 2018, 13, 1301-1328. [CrossRef]

26. Ghazaei, C. Mycobacterium tuberculosis and lipids: Insights into molecular mechanisms from persistence to virulence. J. Res. Med. Sci. 2018, 23, 63. [CrossRef]

27. Gao, T.; Yuan, F.; Liu, Z.; Liu, W.; Zhou, D.; Yang, K.; Duan, Z.; Guo, R.; Liang, W.; Hu, Q.; et al. MnmE, a Central tRNA-Modifying GTPase, Is Essential for the Growth, Pathogenicity, and Arginine Metabolism of Streptococcus suis Serotype 2. Front. Cell Infect. Microbiol. 2019, 9, 173. [CrossRef]

28. Gao, T.; Yuan, F.; Liu, Z.; Liu, W.; Zhou, D.; Yang, K.; Guo, R.; Liang, W.; Zou, G.; Zhou, R.; et al. Proteomic and Metabolomic Analyses Provide Insights into the Mechanism on Arginine Metabolism Regulated by tRNA Modification Enzymes GidA and MnmE of Streptococcus suis. Front. Cell Infect. Microbiol. 2020, 10, 597408. [CrossRef]

29. Yao, J.; Shang, K.; Huang, J.; Ran, W.; Kashif, J.; Wang, L. Overexpression of an ABC transporter and mutations of GyrA, GyrB, and ParC in contributing to high-level ciprofloxacin resistance in Streptococcus suis type 2. Biosci. Trends 2014, 8, 84-92. [CrossRef] 
30. El Garch, F.; Lismond, A.; Piddock, L.J.; Courvalin, P.; Tulkens, P.M.; Van Bambeke, F. Fluoroquinolones induce the expression of pat $\mathrm{A}$ and patB, which encode ABC efflux pumps in Streptococcus pneumoniae. J. Antimicrob Chemother 2010, 65, 2076-2082. [CrossRef]

31. Yang, Y.B.; Chen, J.Q.; Zhao, Y.L.; Bai, J.W.; Ding, W.Y.; Zhou, Y.H.; Chen, X.Y.; Liu, D.; Li, Y.H. Sub-MICs of Azithromycin Decrease Biofilm Formation of Streptococcus suis and Increase Capsular Polysaccharide Content of S. suis. Front. Microbiol. 2016, 7, 1659. [CrossRef]

32. Grauslund, M.; Didion, T.; Kielland-Brandt, M.C.; Andersen, H.A. BAP2, a gene encoding a permease for branched-chain amino acids in Saccharomyces cerevisiae. Biochim. Biophys. Acta 1995, 1269, 275-280. [CrossRef]

33. Liu, K.; Myers, A.R.; Pisithkul, T.; Claas, K.R.; Satyshur, K.A.; Amador-Noguez, D.; Keck, J.L.; Wang, J.D. Molecular mechanism and evolution of guanylate kinase regulation by (p)ppGpp. Mol. Cell 2015, 57, 735-749. [CrossRef]

34. Ye, C.; Bai, X.; Zhang, J.; Jing, H.; Zheng, H.; Du, H.; Cui, Z.; Zhang, S.; Jin, D.; Xu, Y.; et al. Spread of Streptococcus suis sequence type 7, China. Emerg. Infect. Dis. 2008, 14, 787-791. [CrossRef]

35. Gurung, M.; Tamang, M.D.; Moon, D.C.; Kim, S.R.; Jeong, J.H.; Jang, G.C.; Jung, S.C.; Park, Y.H.; Lim, S.K. Molecular Basis of Resistance to Selected Antimicrobial Agents in the Emerging Zoonotic Pathogen Streptococcus suis. J. Clin. Microbiol. 2015, 53, 2332-2336. [CrossRef] [PubMed]

36. Nicholson, T.L.; Waack, U.; Anderson, T.K.; Bayles, D.O.; Zaia, S.R.; Goertz, I.; Eppinger, M.; Hau, S.J.; Brockmeier, S.L.; Shore, S.M. Comparative Virulence and Genomic Analysis of Streptococcus suis Isolates. Front. Microbiol. 2020, 11, 620843. [CrossRef] [PubMed]

37. Roberts, M.M.; Coker, A.R.; Fossati, G.; Mascagni, P.; Coates, A.R.; Wood, S.P. Mycobacterium tuberculosis chaperonin 10 heptamers self-associate through their biologically active loops. J. Bacteriol. 2003, 185, 4172-4185. [CrossRef]

38. Zhang, Y.; Ding, D.; Liu, M.; Yang, X.; Zong, B.; Wang, X.; Chen, H.; Bei, W.; Tan, C. Effect of the glycosyltransferases on the capsular polysaccharide synthesis of Streptococcus suis serotype 2. Microbiol. Res. 2016, 185, 45-54. [CrossRef]

39. Vecht, U.; Wisselink, H.J.; Jellema, M.L.; Smith, H.E. Identification of two proteins associated with virulence of Streptococcus suis type 2. Infect. Immun. 1991, 59, 3156-3162. [CrossRef] [PubMed]

40. Prados de la Torre, E.; Rodriguez-Franco, A.; Rodriguez-Ortega, M.J. Proteomic and Bioinformatic Analysis of Streptococcus suis Human Isolates: Combined Prediction of Potential Vaccine Candidates. Vaccines 2020, 8, 188. [CrossRef] [PubMed]

41. Pian, Y.; Wang, P.; Liu, P.; Zheng, Y.; Zhu, L.; Wang, H.; Xu, B.; Yuan, Y.; Jiang, Y. Proteomics identification of novel fibrinogenbinding proteins of Streptococcus suis contributing to antiphagocytosis. Front. Cell Infect. Microbiol. 2015, 5, 19. [CrossRef] [PubMed]

42. Gao, T.; Tan, M.; Liu, W.; Zhang, C.; Zhang, T.; Zheng, L.; Zhu, J.; Li, L.; Zhou, R. GidA, a tRNA Modification Enzyme, Contributes to the Growth, and Virulence of Streptococcus suis Serotype 2. Front. Cell Infect. Microbiol. 2016, 6, 44. [CrossRef] [PubMed] 